\title{
RECOMENDACIONES TÉCNICAS PARA LA REDUCCIÓN DE PATOLOGÍAS EN EL TERRAZO
}

\author{
(SKILL RECOMMENDATIONS FOR THE REDUCTION OF PATHOLOGIES IN TERRAZO)
}

Sonia Bautista Carrascosa, Ingeniero de Caminos, Canales y Puertos.

Carlos Martín de la Morena, Arquitecto Técnico.

Juan Manuel Mieres Royo, Ingeniero de Caminos, Canales y Puertos.

Dpto. de I+D-NECSO (Entrecanales Cubiertas)

ESPAÑA

Fecha de recepción: 27-II-98

\section{RESUMEN}

El terrazo puede considerarse el material más popular en nuestro país, utilizado como pavimento en edificación. Pero es curioso que después de un largo periodo de tiempo utilizándose, el resultado es un incremento de las patologías, debidas a una mala colocación o selección del pavimento en vez de mejorarse las técnicas de aplicación y selección.

El Departamento de I+D de NECSO (Entrecanales Cubiertas) ha llevado a cabo un proyecto, cuyo principal objetivo es dar una serie de recomendaciones que permitan una reducción de las patologias. Este documento trata de ser un resumen de todo el proceso llevado a cabo desde la fabricación, transporte, gestión en obra y colocación, hasta el mantenimiento.

\section{SUMMARY}

Terrazo can be considered the most popular material used as pavement in buildings in our country. But it is curious that after a long period of use the result is that there is an increase of pathologies instead of having an improvement in its skilled application.

The R\&D Department of NECSO (Entrecanales Cubiertas) has carried out a project which main goal is to give several technical recommendations, in order to reduce the amount of pathologies. This document tries to be a résumé of all processes carried out, including manufacture, transport, in site management, installation and maintenance.
El terrazo ha sido en España, durante muchos años, un elemento básico en la construcción de viviendas. Su relación, precio, prestaciones estéticas y durabilidad han hecho de él un material perfectamente adaptado a los requerimientos de un pavimento.

No obstante, en los últimos años viene produciéndose un aumento de las patologías de este material, patologías que han provocado que muchos arquitectos opten por el pavimento de gres.

Esta actitud, por otra parte muy habitual, de huir de los problemas en vez de analizarlos y solventarlos, nos ha parecido inadecuada, máxime cuando tenemos una reconocida industria del terrazo en nuestro país, que debería servir como base para desarrollar este elemento con nuevas técnicas, más que provocar su desaparición.

Estas reflexiones nos hicieron emprender un proyecto de investigación, acerca del terrazo, que acaba de concluir. En este proyecto se hizo un estudio de las patologías que vienen apareciendo en las obras realizadas en los últimos 20 años por nuestra empresa y aquellas otras de las que teníamos referencia a través de proveedores o bibliografia existente.

Una vez detectadas estas patologías, optamos por reconocer todos y cada uno de los agentes que intervienen en el diseño, la fabricación, el manejo y puesta en obra del terrazo. 
Visitamos fábricas y obras en las que se estaba pavimentando con este material. Hablamos con proveedores, colocadores y pulidores y, por último, analizamos los procedimientos internos de nuestra empresa relacionadas con el terrazo.

Fruto de este trabajo fue un documento que debe de regir todo el proceso de gestión del terrazo, desde su compra hasta terminar con su pulido, abrillantado y entrega al cliente, sin olvidar la fase de proyecto, a la que no se le suele dar la suficiente importancia.

A continuación pasamos a hacer un pequeño resumen del contenido de este documento, que pretendemos se convierta en una guía para los Jefes de obra de nuestra empresa.

\section{1) Tipos de patologías observadas en las baldosas de terrazo}

- Manchas en el centro de la baldosa.

- Manchas o ennegrecido en las proximidades de las juntas entre baldosas.

- Ennegrecido de las propias juntas entre baldosas.

- Decoloración y cambios de tonos en baldosas.

- Juntas abiertas entre baldosas.

- Hundimiento de baldosas con cejas de entre 1 y $2 \mathrm{~mm}$.

- Piedras de la cara vista que saltan.

- Aristas con piedras de la cara vista que saltan.

- Microfisuras.

- Fisuras.

- Eflorescencias.

- Humedad.

- Abombamientos o hundimientos, vistos con luz rasante (aguas).

- Aspereza en la cara vista.

\section{2) Especificaciones técnicas acerca de la gestión de materiales}

Aquí se dan recomendaciones acerca de la recepción, almacenaje y transporte, dentro de la obra, de las baldosas.

Igualmente se hace hincapié en los cementos y arenas, especialmente estas últimas a las que se les da poca importancia y que son las causantes de algunas de las patologías.

La arena sólo se utilizará para mortero, pues queda totalmente prohibida su utilización como capa de nivelación y base sobre la que se coloca el recrecido y el pavimento. La arena utilizada será lavada, con tamaño de grano mínimo $0,08 \mathrm{~mm}$ y máximo $2,5 \mathrm{~mm}$.

La graduación de la arena cumplirá la relación indicada en la tabla I.
TABLA I

\begin{tabular}{|l|c|c|c|}
\hline \multirow{2}{*}{ ARENA } & \multicolumn{3}{|c|}{$\%$ QUE PASA } \\
\hline & LIMITES & \multicolumn{2}{|c|}{ LIMITES ADICIONALES } \\
\cline { 3 - 4 } & TOTALES & C & \\
\hline $2,36 \mathrm{~mm}$ & $60-100$ & $60-100$ & \\
\hline $1,18 \mathrm{~mm}$ & $300-100$ & $30-90$ & \\
\hline $600 \mu \mathrm{m}$ & $15-100$ & $15-54$ & \\
\hline $300 \mu \mathrm{m}$ & $5-70$ & $5-40$ & \\
\hline $15 \mu \mathrm{m}$ & $0-15^{(1)}$ & -- & - \\
\hline
\end{tabular}

(1) Hasta $20 \%$ en roca machacada.

Esta afirmación es el resultado final de una amplia investigación que contempla, en primer lugar, la normativa del Reino Unido y, en segundo, la francesa, contrastándolas con la normativa española. La primera prescinde de la capa de arena mientras que la segunda, aunque la permite, exige unos controles tan exhaustivos en cuanto al empleo de una adecuada granulometría, compactación y ejecución de la colocación del pavimento, que resulta, en cierto modo, una manera indirecta de limitar su empleo.

En la normativa española, sin embargo, "Norma Tecnológica de la Edificación NTE-RSR. Revestimiento de Suelos. Piezas Rígidas", que no es de obligado cumplimiento, pero constituye una guía práctica en el proceso español de la edificación, se indica la conveniencia de la colocación de la capa de arena.

Finalmente, analizando nuestra estadística, observamos cómo actualmente se producen numerosas patologías en los pavimentos de piezas rígidas, favorecidas, en unos casos, por el aumento de las dimensiones de las propias baldosas y, en otros, por su empleo en zonas de elevado tránsito. En nuestros laboratorios se ha llegado a la conclusión de que la colocación de la capa de arena sin una cuidadosa granulometría y correcta compactación puede ocasionar desplazamientos y asientos diferenciales que las piezas de pavimento, de mayor tamaño, no pueden absorber, produciéndose, en estos casos, desniveles, hundimientos, cejas y roturas que nos hacen cuestionar su empleo. Por eso, en evitación de mayores complicaciones, se prefiere eliminar el empleo de la arena como capa niveladora y base sobre la que se coloca el recrecido y el pavimento, obligando al empleo de mortero para estabilizarla y colocando, además, una lámina separadora (polietileno, geotextil, etc...), para conseguir el efecto desolidarizador. 


\section{3) Preparación de trabajos, materiales y componentes}

En este punto, se hacen recomendaciones acerca de los conductos y tuberías colocadas bajo el pavimento, la iluminación durante la colocación, preparación de los morteros, cotas y unas condiciones generales, entre las que cabe destacar:

a) Solamente se debe extender mortero, en tal cantidad, que proporcione trabajo para, aproximadamente, 2-3 horas.

b) Durante la colocación de las baldosas, las áreas colocadas no deberán ser pisadas por nadie, excepto por los colocadores, y tomando precauciones, como pisar sobre tableros de madera que distribuyan los pesos.

c) Igualmente importante es que el pavimento recién acabado no sea sometido al tráfico, ya sea peatonal o de vehículos, antes del fraguado del mortero y de que se haya formado una unión suficiente entre forjado y mortero, creándose, a tal efecto, un calendario de uso, según los tipos de tráfico a soportar.

d) Como referencia, se puede afirmar que el tráfico, exclusivamente peatonal, se puede permitir después de 4 días del acabado. Se debe esperar por lo menos dos semanas antes de permitir tráficos más intensos.

e) La superficie de la obra siempre debe permanecer limpia y exenta de cementos y todo tipo de material que pudiera provocar manchas e, incluso, extender serrín de madera blanca para su protección.

f) Para todo tipo de trabajo con materiales a base de aceites, grasas, pinturas, etc., ejecutados sobre el pavimento, éste se debe cubrir temporalmente, sin que esto impida el secado del pavimento y provoque la aparición de manchas en el material, por lo cual insistimos en la utilización de serrín, en cuyo caso sirve, además, de material absorbente de humedad.
Si se utiliza maquinaria pesada que pudiera dañar las baldosas, se recomienda tapar el suelo con tablones o planchas de madera.

g) Donde no existan los riesgos anteriores, basta con una capa de serrín blanco, que no manche, para obtener la protección necesaria hasta que el resto de operaciones del edificio concluyan.

No obstante, bajo nuestro punto de vista, una de las aportaciones más significativas se aprecian en la tabla II, en donde, partiendo de dos posibilidades: forjado de vigueta y bovedilla o de losa, se dan unos plazos para todas las operaciones que completan la colocación del terrazo. Si se respetan estos plazos, es seguro que un porcentaje muy alto de las patologías conocidas, desaparecerá.

Esta tabla ya nos hace pensar en la conveniencia de recomendar no realizar forjados unidireccionales, cuya tipología, por otra parte, en la Comunidad Europea va camino de extinguirse debido a la problemática que ocasiona su elevada flexibilidad, así como la necesaria ejecución de las edificaciones a un ritmo mucho más lento que el que las actuales necesidades de contratación de obra en plazos de 16-18 meses permiten.

Por ello, con más frecuencia cada vez, se tiende a construir los forjados a base de losa continua, con un espesor de $22 \mathrm{~cm}$ y mucho más rígidos, posibilitando la correcta pavimentación al ritmo que requieren los actuales plazos de obra.

\section{4) Colocación de las baldosas de terrazo}

Antes de colocar las baldosas, se debe de haber finalizado los cerramientos y han de estar terminados los tabiques y los guarnecidos o enfoscados interiores, colocados los vidrios, tuberías y canalizaciones de todo tipo. La pintura se ejecutará después de la terminación del pavimento.

\begin{tabular}{|c|c|c|c|c|c|c|c|c|c|c|c|c|c|c|c|c|c|}
\hline & & \multicolumn{2}{|c|}{ 21-25 SEMANAS (5-6 MESES) } & & & & & & & & & & & & \multicolumn{2}{|c|}{14 DiAS } & \\
\hline & & & $\begin{array}{c}8 \\
\text { SEMANAS }\end{array}$ & & 3.5 DIAS & & 3.5 DIAS & & 3 DiAS & & 2 DIAS & & 5.7 DIAS & & 4 DIAS & & \\
\hline 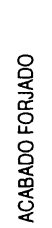 & 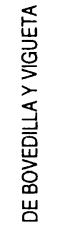 & 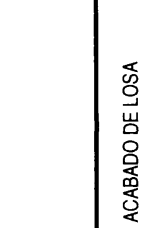 & & 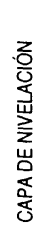 & & 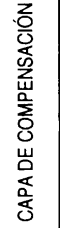 & & 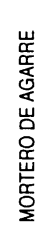 & & $\begin{array}{l}\text { 呈 } \\
\text { 䓌 } \\
\text { 壱 }\end{array}$ & & 吕 & & 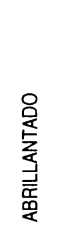 & & 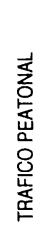 & 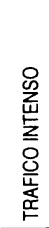 \\
\hline
\end{tabular}

Nota: En vivienda, el 90\% de los forjados se hacen con vigueta/bovedilla. Con reticular sólo P. Comerciales y Garajes. 
Según el tipo de tráfico que vaya a soportar el pavimento, el agarre de las baldosas será diferente.

A continuación describimos las dos posibilidades:

\section{A)Tráfico peatonal}

Se distinguen dos tipos de agarre, según el estado del soporte

\section{1) El soporte cumple con las tolerancias admitidas}

En este caso, el lecho consiste en una lámina de polietileno u otra lámina impermeable, puesta directamente sobre el soporte, con un solape $>10 \mathrm{~cm}$. La lámina será de 150 micras (Fig. 1).

En ningún caso el espesor de la capa de mortero de agarre o recrecido de piso debe exceder los $3 \mathrm{~cm}$, ya que se retrae al fraguar y si es de un espesor mayor, la retracción provocaría patologías.

Por lo tanto, si para alcanzar el nivel previsto de pavimento terminado se necesitan más de $3 \mathrm{~cm}$ de mortero, encima de la lámina se colocará una capa de compensación previa de mortero. Esta capa se dejará fraguar durante 3-5 días.

La capa de mortero de agarre propiamente dicha tendrá un espesor que puede oscilar entre 1,5 y $3 \mathrm{~cm}$, siendo el espesor ideal $2 \mathrm{~cm}$.

Tanto la capa de mortero de agarre como la de compensación, si fuera necesaria, irán separadas de todas las tabiquerías interiores, dejando una junta perimetral en todo su espesor, la cual puede estar previamente preparada, adosando a toda la tabiquería una banda de cualquier elemento flexible, pero que sea capaz de soportar los esfuerzos laterales del recrecido de mortero. El espesor será, como mínimo, de $5 \mathrm{~mm}$. Esta junta también se colocará en el encuentro entre habitaciones, pasillo y en toda aquella dimensión que exceda de $6 \mathrm{~m}$.

Encima de la capa de agarre se colocarán directamente las baldosas, dejando entre ellas una junta de 2 a $3 \mathrm{~mm}$ de anchura y, de al menos, $5 \mathrm{~mm}$ en todo el perímetro de las habitaciones, junta que, después de ir convenientemente sellada, quedará tapada por el rodapié, el cual irá sólo recibido al paramento vertical y sellado elásticamente en el encuentro con el solado de terrazo.

\section{2) El soporte no cumple con las tolerancias admitidas}

En este caso hace falta, en primer lugar, nivelar el soporte, para que se pueda colocar la lámina de polietileno sin peligro de deteriorarla (Fig. 2).

La capa de nivelación consistirá en el mismo material que el mortero de agarre.

Se dejará fraguar durante 3-5 días.

El espesor de esta capa oscilará entre 2,5 y $4 \mathrm{~cm}$ y no se excederá, en ningún caso, de $4 \mathrm{~cm}$, por aumentar el riesgo de pérdida de adherencia con el forjado.

En el caso de existir conducciones embebidas se reforzará la zona con un mallazo.

Una vez que la superficie del soporte está nivelada y lisa, se sigue con la misma estructura que en el caso anterior.

El mortero, sin embargo, tendrá consistencias diferentes según la capa: la inferior, de compensación, deberá tener consistencia de tierra húmeda (semi-seca); la superior, consistencia plástica.

Así, el agua sobrante de la capa superior proporcionará la cantidad necesaria para la hidratación completa de la capa inferior.

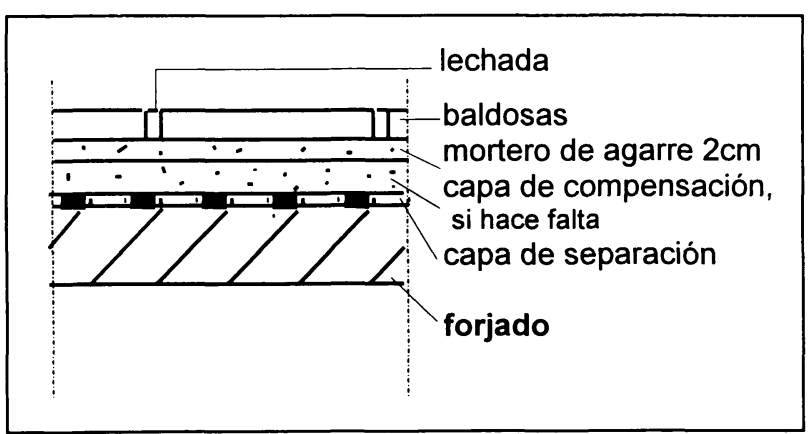

Fig. 1.- Forjado que cumple tolerancias.

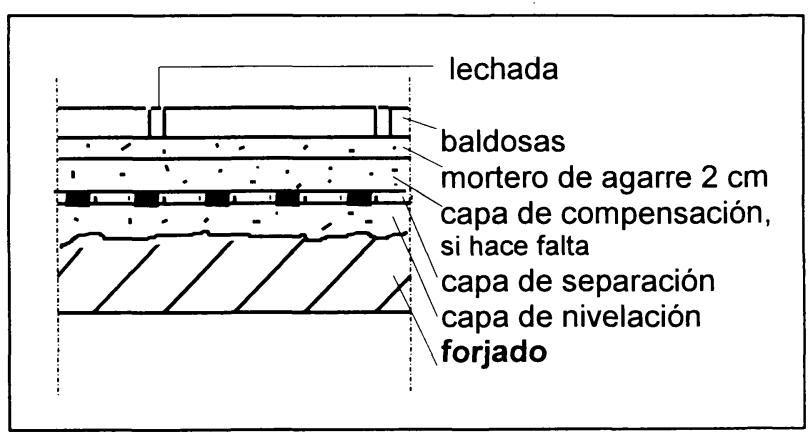

Fig. 2.- Forjado que no cumple tolerancias. 


\section{B)Tráfico pesado}

En el caso de preverse tráfico intenso o pesado, o bien cuando se esperan condiciones de servicio rigurosas, se debe asegurar una buena adherencia entre esta capa y el forjado o soporte, de forma que se garantice que las dos capas trabajan conjuntamente.

\section{Técnica con mortero semi-seco}

Si se realiza la capa de agarre con mortero semi-seco, para garantizar la adherencia se utiliza como agente soldador lechada de cemento (gris o blanco) (Fig. 3).

Esta lechada de cemento puro se extenderá sobre la superficie 30 minutos antes de extender el mortero de agarre. Varias horas antes del extendido de la lechada se mantendrá la superficie convenientemente húmeda.

Para que este agente trabaje correctamente se debe de preparar la superficie de apoyo (base) mediante un raspado adecuado, dejando tras una limpieza el árido visto, sin dejar material suelto. Esto se puede prever en la ejecución del forjado y dejar éste con una cierta rugosidad. Esta condición permitirá una mayor efectividad de la unión entre capas.

La relación cemento:arena no será mayor de 1:3 ni menor de $1: 4$, en peso.

La relación agua/cemento se mantendrá entre 0,55 y 0,6 teniendo en cuenta la humedad de la arena.

La capa de mortero se extenderá con un espesor aproximadamente del 10 al $15 \%$ por encima del espesor final previsto, que se conseguirá mediante la compactación con maceta de goma.

Inmediatamente después del mortero se extenderá un "slurry" cemento:arena de 1:1 y de $2 \mathrm{~mm}$ de espesor sobre el que se colocarán las baldosas.

\section{Técnica con mortero plástico}

Si se realiza la capa de agarre con mortero plástico, para garantizar la adherencia se debe preparar la superficie de apoyo mediante un raspado adecuado, dejando, tras una limpieza, el árido visto. El propio mortero plástico deberá servir de agente de unión (Fig. 4).

La capa de mortero tendrá una relación cemento:arena de 1:3-1:4, con consistencia plástica, debiendo compactarse a continuación, hasta alcanzar una densidad de $1.900-2.150 \mathrm{~kg} / \mathrm{m}^{3}$. La correcta densidad se comprobará in situ.

La capa de mortero de agarre propiamente dicha tendrá un espesor que puede oscilar entre 1,5 y $3 \mathrm{~cm}$, siendo el espesor ideal $2 \mathrm{~cm}$.

Si por motivos de nivelación del forjado se recurriera a efectuar una capa de nivelación, ésta se ejecutará conforme a las especificaciones anteriores.

Encima de la capa de agarre se espolvoreará cemento antes de la colocación de las baldosas.

\section{5) Juntas}

Muchas de las patologías aparecen por un mal diseño de juntas. El arquitecto, en sus proyectos, debería incluir un estudio de juntas del pavimento, siempre siguiendo las siguientes recomendaciones:

Entre baldosa y baldosa, se dejará una junta de 2-3 mm (nunca menor de $2 \mathrm{~mm}$ ).

Se colocarán juntas de movimiento reforzadas con flejes:

- cada 6 metros (cada 3 baldosas, en caso de tráfico pesado).

- si la superficie excede los $40 \mathrm{~m}^{2}$.

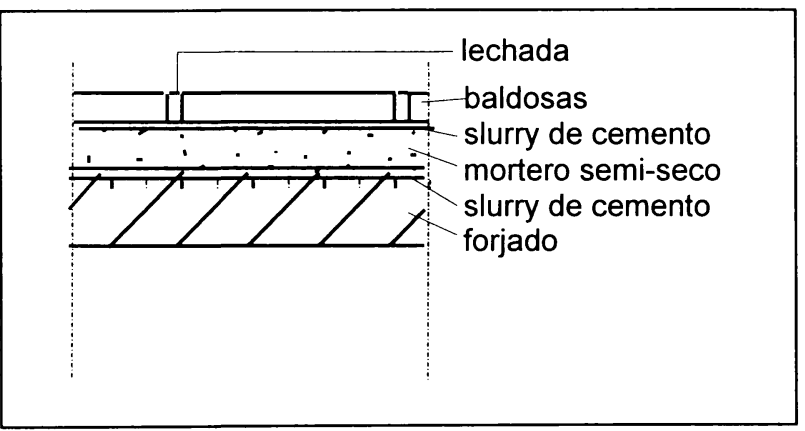

Fig. 3.- Técnica de mortero semi-seco.

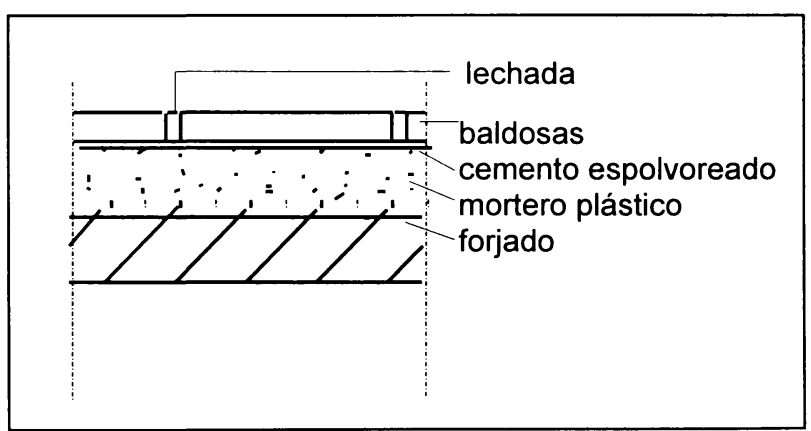

Fig. 4.- Técnica de mortero plástico. 
- respetando las juntas de dilatación estructurales.

- encima de muros de carga, vigas, etc.

- en el encuentro con pilares, tabiques, etc.

Además, se dejará una junta perimetral de $5 \mathrm{~mm}$ de anchura.

\section{6) Pulido y abrillantado}

Por último, operaciones como enlechado y descejado, pulido y abrillantado son fundamentales a la hora de tapar poros, pero, a su vez, permitir, antes de que esto ocurra, la salida de la humedad hacia el exterior, evitando las manchas oscuras en las juntas. Por ello, la correcta selección inicial de piezas en cuanto a su coloración es esencial para evitar cualquier imperfección posterior.

En cuanto a la ejecución, el documento distingue tres fases:

a) Enlechado y descejado

A los 3 días, como pronto, de colocar las baldosas, se procederá al enlechado.

El enlechado estará formado por cemento blanco tipo 45, árido microtriturado y pigmentos. Una vez amasada la mezcla, será utilizada antes de 30 minutos. Extendida y perfectamente rellenas las juntas, se retirará el sobrante con un cepillo de banda de goma.

La lechada se dejará fraguar, como mínimo, 2 días y durante este período se evitarán condiciones extremas de curado.

Antes de proceder al descejado se realizará una limpieza previa del sobrante de lechada con estropajo de esparto. A continuación se comenzará el descejado con la corona de piedra de carborundum, de grano $n^{\circ} 60$ u 80, según el aspecto de las cejas.

El rendimiento con esta corona será, aproximadamente, de 5 a 6 minutos $/ \mathrm{m}^{2}$ y no deberá nunca ser mayor, ya que implicaría un pulido deficiente, con consecuencias no deseadas. A continuación se limpiará y lavará el suelo sólo con agua.

Seguidamente se dará el empaste; como líquido se utilizará el mortero de cemento suministrado por el fabricante (nunca se deberá usar lechada de cemento sin áridos). Como corona se utilizará, según el estado del piso, la piedra de grano $\mathrm{n}^{\circ} 100$ ó 120 . El rendimiento será, de nuevo, aproximadamente de 5 a 6 minutos $/ \mathrm{m}^{2}$.

El siguiente paso consiste en una buena limpieza del suelo sólo con agua.

\section{b) Pulido}

Previamente será definido por el Director de Obra o por el Jefe de Obra el grado final de pulido, correspondiente a las coronas con abrasivo $\mathrm{n}^{\text {os }} 220,400$ u 800 (p.e.).

Entre la última corona utilizada en el descejado y la final a la que queremos llegar con el pulido, se intercalarán las piedras $\mathrm{n}^{\text {os }} 200 / 220,360 / 400,600 / 650 \mathrm{y}$, finalmente, la $\mathrm{n}^{\circ} 800$

Normalmente será suficiente con llegar a un pulido con piedra de grano $n^{\circ} 400$.

El rendimiento de cada una de estas piedras será de 2 a 3 minutos $/ \mathrm{m}^{2}$.

\section{c) Abrillantado}

El piso será lavado con agua antes de proceder al abrillantado, dejando a su vez que la baldosa seque. Se dejará pasar un mínimo de 5 a 7 días desde la finalización del pulido hasta el comienzo del abrillantado, según la zona geográfica y según la estación climatológica.

Se darán al menos 2 pasadas con el disco de lana de acero y una solución líquida de fluoxilicato de magnesio y ácido fosfórico, de cualquier marca comercial.

Para facilitar el secado del suelo se recomienda dar 2 ó 3 pasadas con serrín de madera blanca (de pino o chopo), de forma que se elimine el máximo de humedad, al absorberla el serrín.

A continuación se darán 1 ó 2 manos de cera neutra, de marca comercial.

Es importante saber que el pulido y el abrillantado cierran el poro de la baldosa, haciéndola impermeable e impidiendo la penetración de la suciedad, pero impidiendo, a su vez, que salga la humedad residual acumulada durante todo el proceso anteriormente descrito, por lo que la recomendación de "baldosa seca" durante todo el proceso es fundamental.

Por último, se dan recomendaciones de mantenimiento y conservación, donde se incluyen las protecciones durante la ejecución del resto de operaciones hasta la entrega del edificio al cliente.

\section{7) Gestión de compras y contratación de colocadores y pulidores}

En este artículo no incluimos, por su extensión, una parte dedicada a la evaluación de fábricas, en donde se analizan consideraciones como:

- Tolerancias de dimensiones de baldosas. 
- Espesores de la cara vista.

- Espesores de la capa de base.

- Capacidad de fabricación.

- Capacidad de buen suministro.

- Organización dentro de la fábrica.

- Transporte de los áridos, lavados en cantera.

- Organización de los áridos y las arenas (protección de acopios).

- Lavado de los áridos en fábrica, previa a su utilización.

- Análisis periódico de las aguas que se utilizan para la confección de las masas de mortero y hormigón.

- Tipo de máquina con la que fabrican y antigüedad.

- $\mathrm{N}^{\mathrm{o}}$ de estaciones de vibrado y frecuencia de vibración.

- $\mathrm{N}^{0}$ de estaciones de prensado y atmósferas de presión.

- Curado de baldosas, cuidados.

- Cámara húmeda, control de temperatura y humedad.
- Tiempo de curado

- Tiempo de desecación y fraguado, previo al pulido en fábrica.

- Máquina pulidora.

- $\mathrm{N}^{\circ}$ de coronas que utiliza para el desbastado y pulido.

- $N^{\circ}$ de abrasivos utilizados en fábrica.

- Forma de colocación de las baldosas sobre el palé.

- Protección entre baldosas.

- Protecciones que lleva el palé (plastificado o esterilla).

- Lugar de acopio en fábrica. Cubierto y protegido del sol o de las heladas.

El proyecto de investigación ha concluido con la redacción de unas guías para la elaboración de pedidos de compras y selección de colocadores y pulidores.

En definitiva, hemos tratado de hacer un análisis del terrazo en su conjunto, buscando las causas que han producido el aumento espectacular de las patologías y no nos cabe ninguna duda que siguiendo el documento generado por el Departamento de I+D de NECSO, éstas deben
reducirse.

\section{publicación del IETCC/CSIC}

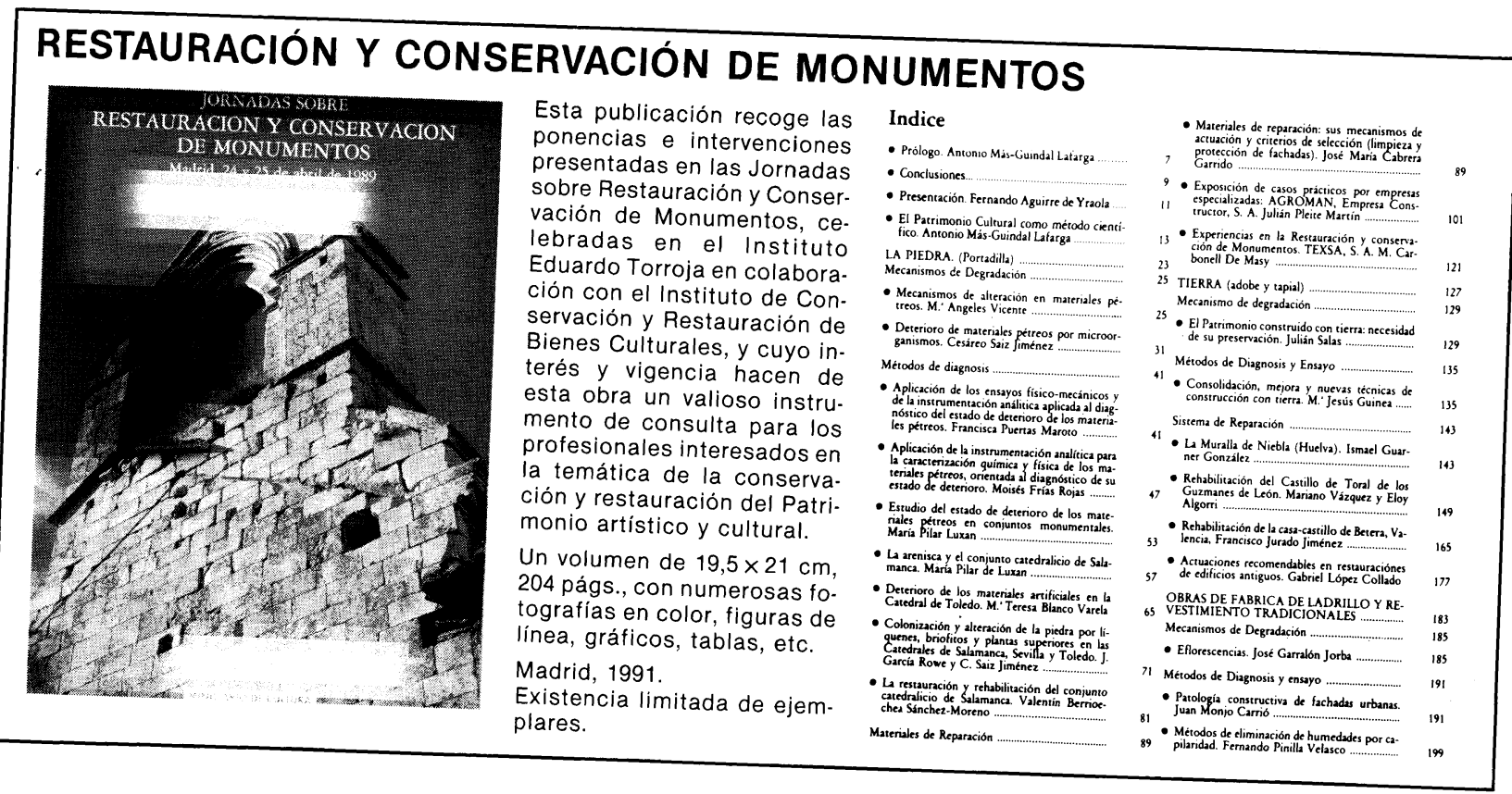

